\title{
A Boundary Localisation Algorithm Consistent with Human Visual Perception
}

\author{
E. Claridge, School of Computer Science, The University of Birmingham, Birmingham B15 2TT, U.K.
}

\begin{abstract}
This paper describes an algorithm, based on psychophysical models of edge localisation, for computing an edge location in presence of blur. The algorithm successfully finds the most likely location at which a human observer would place the edge. For simulated image data the maximum displacement between the boundary placing by the human subjects and by the algorithm was 1.7 pixels. The application of the algorithm to real medical images also showed good agreement, with average radial displacement of 3.3 pixels for skin lesions and 2.7 pixels for mammographic lesions. As the algorithm is based on general principles believed to underlie human visual perception it should be generally applicable.
\end{abstract}

\section{Introduction}

It is now well accepted in the computer vision community that the problem of mathematically optimal edge detection operators has been solved (e.g. [12]). These operators work in the signal domain and seek to find the optimally-fitting underlying edge model at an appropriate spatial scale and orientation and then to detect in it the location of the maximum gradient, usually found by computing the location of the zero crossing of the second spatial derivative of image luminances. Operators of this nature are also accepted as plausible models for edge detection in biological visual systems. In their influential paper "Theory of edge detection" Marr \& Hildreth [9] demonstrated that the results produced by the zero-crossing algorithm are in good agreement with the results of psychophysical experiments by Wilson and Bergen [16] and of physiological research by Dreher \& Sanderson [3].

Direct application of the zero-crossing algorithm to a blurred edge will not usually indicate the same location which a human observer would choose. Helmholtz (quoted in [10]) observed that bright areas on a dark background appear larger than they really are. This so-called "irradiation effect" can be explained by the non-linear saturating (logarithmic) response of retinal receptors. If the input intensity is first transformed in this way then the zero crossing of the second derivative of the resulting signal will be shifted towards darker part of the edge [10]).

The irradiation effect is a perceptual phenomenon and, as such, it does not challenge the choice of the zero- crossing as the correct position of edge location. In most cases edge localisation is performed in the context of interpretation of optical images of the natural world. In such images an edge is taken to be the location of discontinuity in image luminance which corresponds to discontinuity in depth, orientation or reflective properties of 3D surfaces in the real world. Whereas most 3D discontinuities are well defined and abrupt, their images (both retinal and digital) are often not. Distorting factors include noise and blur caused by the viewing conditions (e.g. light scatter) and by the imaging apparatus (e.g. low spatial resolution, incorrect focus, motion unsharpness, receptor noise). Thus any unsharpness at the image boundary is a distortion of the real, sharp edge, whose boundary normally coincides with the zero-crossing.

However, the zero-crossing model is not appropriate for all imaging conditions. Examples from the medical domain include projective images (e.g. x-ray), images showing partial volume effect (most tomographic images such as CT or MRI) and images of translucent surfaces (e.g. skin). For such images the blur present at the object periphery is not necessarily a "distortion of a real sharp edge" but it most likely depicts the edge property such as gradual decrease in thickness, in tissue density, or in pigment concentration. Clearly, the zero-crossing model of the edge localisation will not be applicable here; the boundary location will be computed within the area of the projected object rather than at its periphery (e.g. see Figure 4 (b)).

If it is accepted that the zero-crossing model is inappropriate, what could provide the basis for the design and evaluation of computer algorithms for this type of image, especially if the physical "ground truth" is difficult or impossible to obtain? One possibility is through modelling of the physical processes of image formation: having developed a forward imaging model an inverse solution is sought. This approach is fraught with difficulties as the imaging process always reduces the dimensionality of data. Progress is being made in the areas of $\mathrm{x}$-ray mammography [6]) and skin imaging [2]).

Another commonly used approach is to take as the "ground truth" the edges located by human observers. Although this perceived boundary location is inferior to the physical location, it has at least one merit. As it reflects the human visual perception, any geometric descriptions of objects thus delineated can be directly related to the existing clinical descriptions. Obtaining the model data in this 
way is not trivial. If $\mathrm{N}$ observers provide $\mathrm{N}$ edge locations for an object, how should these locations be combined? Simple statistical functions such as, for example, average or median location, are not entirely satisfactory because the computed location may fall at a position not chosen by any observer (e.g. see the outline in figure 4 (c) at the " 4 o'clock" location).

A more plausible solution might be to consider models based on results of research in psychophysics. These models often take the form of functions specifying responses for a given class of visual stimuli and are based on statistics from experiments involving many subjects. Although their source is also experimental data, they have two advantages over the statistics derived from the explicit edge location data for a particular data set. First of all, the models embody explanation of the processes described by the functions, often obtained and verified through several different experimental techniques and supported by physiological data. Secondly, the statistics are calculated in the space of function parameters and not in the space of physical point locations, which makes them general.

This paper describes an algorithm for computing edge location based on psychophysical models of edge localisation in humans. For objects with blurred peripheries the algorithm finds the most likely location at which a human observer would place the edge. Section 2 summarises relevant aspects of the human visual perception as documented in psychophysical literature. Section 3 describes the computational model. Section 4 compares performance of the algorithm against the human data. Results are provided for simple simulated images and for real medical images, including pigmented skin lesions and mammographic lesions. A discussion in section 5 concludes the paper.

\section{Edge localisation in humans}

A number of psychologists studying visual perception investigated the accuracy of edge localisation under conditions of varying blur and varying contrast. Research by Watt \& Morgan [14], Mather and Morgan [10], Morgan et al. [11] and Georgeson \& Freeman [4] clearly show that for blurred 1-dimensional edges there is a shift in perceived edge location away from the zero crossing. The magnitude of the shift increases with the increase in magnitude of both the edge blur and edge contrast. Their proposed model of the shift is based on non-linearity of perceived brightness, with the saturation function (1) where $c$ is a constant:

$$
\mathrm{R}=\frac{\mathrm{I}_{\mathrm{L} \supseteq \mathrm{R}_{\text {max }}}}{\mathrm{I}_{\mathrm{L}}+c \supseteq \mathrm{I}_{\mathrm{Lmax}}}
$$

If the input luminance $\mathrm{I}_{\mathrm{L}}$ is transformed through this function, the edge will be perceived at the location of zero- crossing of the output (response) function R.

Claridge \& Jefferies [1] extended investigations to 2dimensional stimuli with different polarities. For objects brighter than their background their finding were similar to those of Mather, Morgan and Georgeson op cit. but for stimuli of the opposite polarity (objects darker than their background) the shift decreased with increase in contrast. Figure 1 shows the shift in perceived edge location (in pixels, 1 pixel = 2 min arc) as a function of blur, contrast and polarity for a general case of 2-dimensional stimulus.

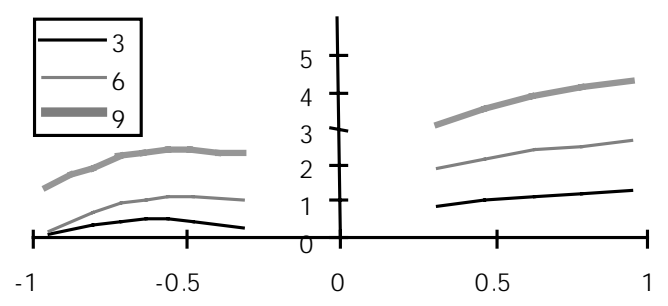

Figure 1. Shift in perceived edge location based on results of psychophysical experiments for blur magnitudes 3, 6 and 9. Magnitude of contrast is shown along $x$-axis and the perceived shift on y-axis.

As the shift is a function of blur and contrast the models of blur and contrast perception need to be incorporated into the model of edge localisation. The commonly used measure of magnitude of perceived contrast is Michelson contrast $\mathrm{C}$ defined as:

$$
\mathrm{C}=\frac{\mathrm{I}_{\mathrm{Lmax}}-\mathrm{I}_{\mathrm{Lmin}}}{\mathrm{I}_{\mathrm{Lmax}}+\mathrm{I}_{\mathrm{Lmin}}}
$$

The magnitude of perceived blur $\sigma$ can be computed as proposed by Georgeson [5]:

$$
\sigma=\sqrt{-\frac{\mathrm{I}^{\prime}\left(\mathrm{x}_{\mathrm{zc}}\right)}{\mathrm{I}^{\prime \prime}\left(\mathrm{x}_{\mathrm{zC}}\right)}}
$$

where for point $x_{\mathrm{ZC}} I$ I" $\left(\mathrm{x}_{\mathrm{zC}}\right)=0$ (i.e. $\mathrm{x}_{\mathrm{zC}}$ is the zerocrossing of the second derivative for edge profile $\mathrm{I}(\mathrm{x})$ ). The absence of any scaling parameters suggests that blur perception is invariant with respect to the edge contrast. Similar results were reported by Morgan et al. [11].

\section{Computational model}

The psychophysical models of edge localisation, contrast and blur perception outlined above provide a basis for the algorithm which computes the perceived edge location for 2-dimensional objects with blurred peripheries. Prior to calculations an object is decomposed into a set of 1dimensional radial profiles by applying a polar coordinate 
transform. For each radial profile the perceived edge location $\mathrm{X}_{\mathrm{PE}}$ is determined by calculating the displacement $\Delta \mathrm{x}$ with respect to the location of zero crossing $\mathrm{x}_{\mathrm{zc}}$. The magnitude of the displacement is a function of contrast $\mathrm{C}$ and blur $\sigma$ at $\mathrm{x}_{\mathrm{zc}}$. Thus:

$$
\mathrm{x}_{\mathrm{PE}}=\mathrm{x}_{\mathrm{ZC}}+\Delta \mathrm{x}\left(\mathrm{C}\left(\mathrm{x}_{\mathrm{ZC}}\right), \sigma\left(\mathrm{x}_{\mathrm{ZC}}\right)\right)
$$

Contrast is calculated using formula (2), where the minimum and maximum values $\mathrm{I}_{\mathrm{Lmin}}$ and $\mathrm{I}_{\mathrm{Lmax}}$ correspond to the matched step edge, as shown in Figure 2 (top). The blur parameter is derived by deconvolving the edge profile with the matched step edge (Figure 2 (bottom)). The result of deconvolution is a profile with an approximate Gaussian shape from which the parameter sigma is estimated [13].

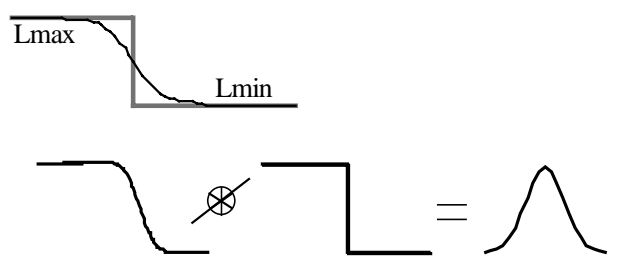

Figure 2. Top: A blurred edge and the matched step edge; Bottom: A blurred edge is deconvolved with the matched step edge, resulting in a Gaussian curve.

\section{Results}

The algorithm was first verified on the experimental image data and then tests were carried out on real image data where the objects were lesions in two different types of medical images.

\subsection{Simulated images}

The objective of verification was to compare experimental results with the results of the algorithm using a common set of simple computer-generated stimuli with known parametrisation. Images used for verification were circular blobs of 40 pixel in radius with Gaussian-blurred peripheries, placed on mid-grey background. Blur parameter $\sigma$ was set to 3, 6 and 9 and contrast parameter $C$ to $31 \%, 63 \%$ and $94 \%$ for both polarities. In total 18 images were used. Fourteen subjects took part in the experiment, marking a position of the perceived boundary along four radial lines from the blob centre outwards. This was performed twice for each of the eighteen blobs [7]). The positions, recorded in the form of the radial distance, were compared with the positions calculated by the computer algorithm. The results shown in Table 1 indicate good correspondence.

\begin{tabular}{|l|l|l|l|}
\hline & $\sigma=3$ & $\sigma=6$ & $\sigma=9$ \\
\hline
\end{tabular}

\begin{tabular}{|l|c|c|c|}
\hline $\mathrm{C}=+31 \%$ & 0.3 & 0.9 & 0.6 \\
\hline $\mathrm{C}=+63 \%$ & 0.7 & 0.9 & 1.7 \\
\hline $\mathrm{C}=+94 \%$ & 0.0 & 1.3 & 1.6 \\
\hline $\mathrm{C}=-31 \%$ & -0.2 & -0.3 & 0.1 \\
\hline $\mathrm{C}=-63 \%$ & 0.2 & 0.4 & 0.8 \\
\hline $\mathrm{C}=-94 \%$ & 0.1 & 0.1 & 0.5 \\
\hline
\end{tabular}

Table 1. The difference (in pixels) between the boundary placing by the human observers and as calculated by the computer algorithm. Contrast parameter for bright blobs is signed with "+" and for dark blobs with "-".

\subsection{Lesions in medical images}

The algorithm was applied to calculate the positions of the perceived boundary of lesions in two different types of medical images: photographic images of skin lesions and $\mathrm{x}$-ray mammograms. Skin lesions appear as dark blobs on bright background (skin) and mammographic lesions appear as bright blobs on dark background. In both types of lesions contrast and peripheral blur may vary. For these real images the exact position of the boundary is not available. The objective here was to compare the boundary location predicted by the algorithm with the locations indicated by human observers.

Pigmented skin lesions. The data set comprised images of 12 skin lesions. Eighteen subjects (clinicians) took part in the experiment. They were asked to draw a boundary around each lesion. The outline data was digitised, converted from Cartesian to polar coordinates and recorded as the radial distance between the centre of gravity of the lesion and the marked outline (256 points for each lesion). Simple statistics characterising the agreement between the outlines were calculated and are shown in Table 2 (HS) and Figure 3. For the same image set the boundary positions were calculated by the computer algorithm. The algorithm includes a self-testing component which enables it to reject erroneous results. The data derived to calculate the perceived edge location $\left(\mathrm{x}_{\mathrm{zc}}, \mathrm{I}_{\mathrm{Lmin}}, \mathrm{I}_{\mathrm{Lmax}}\right.$ and $\sigma$ ) is used to reconstruct the underlying edge profile. If the error between the original and the reconstructed edge exceeds the user specified threshold, the parametrisation is considered incorrect and the results deemed invalid. About $25 \%$ of the data points were rejected in this way. The valid points were compared with the outlines drawn by the subjects. As those outlines suffered from inconsistencies, the comparisons were made only for those boundary positions where more than 5 respondents agreed on the boundary location. Table 2 (HS-C-SL) summarises the results of the comparison. The figures without brackets refer to the absolute radial distance (in pixels) between the respective boundary points. The figures in brackets show the results for signed 
distances, where the negative values indicates that the computer-derived boundary was placed nearer the centre of the lesion than the clinical boundary.

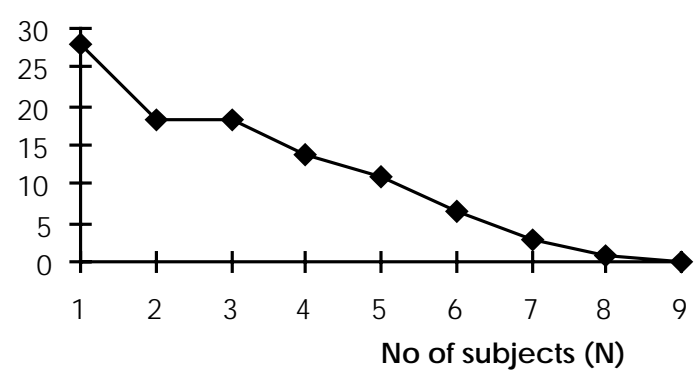

Figure 3. The percentage of all the boundary points for which $\mathbf{N}$ subjects selected the same boundary location.

Mammographic lesions. The data set consisted of $36 \mathrm{im}-$ ages representing 18 lesions (for each lesion two x-ray images were taken at different angles). One consultant radiologist took part in the experiment and was asked to draw a boundary around each lesion. As in the previous experiment, the outline data was digitised, converted to polar coordinates and recorded as the radial distance between the centre of gravity of the lesion and the marked outline, giving 256 points for each lesion.

For the same image set the boundary positions were calculated by the computer algorithm. About $15 \%$ of the data points were rejected as unsuitable for analysis. The valid points were compared with the outlines drawn by the subject. Table 2 (HS-C-ML) summarises the results of the comparison.

\begin{tabular}{|c|c|c|c|c|}
\hline & $\min$ & $\max$ & average & st. dev. \\
\hline HS & 5 & 20 & 11.97 & 3.04 \\
\hline HS-C-SL & 0 & 8 & 3.59 & 2.23 \\
& $(-4)$ & $(8)$ & $(3.32)$ & $(2.63)$ \\
\hline HS-C-ML & 0 & 14 & 2.75 & 2.38 \\
& $(-14)$ & $(9)$ & $(0.67)$ & $(3.57)$ \\
\hline
\end{tabular}

Table 2. The statistics characterising the degree of correspondence between the outlines drawn by the subjects (HS), between the outlines drawn by the subjects and those derived by the computer algorithm for skin lesions (HS-C-SL) and for mammographic lesions (HS$\mathrm{C}-\mathrm{ML}$ ). The figures refer to the radial distance (in pixels) between the extreme clinical markings for individual boundary points.

\subsection{Discussion}

The results of the comparisons show very good agreement between the boundary placing of the human subjects and those obtained by the computer algorithm. The results on simulated data, covering a broad range of blur and contrast parameters, showed a maximum error of 1.7 pixels, with average error of 0.59 . This means that in the noisefree experimental conditions the algorithm can be considered to be an appropriate model of the boundary placing consistent with the human visual perception. It should be noted that the computer model was built on the basis of different experimental data than that used for the experiments in this paper.

The application of the algorithm to real medical images provided further confirmation of its predictive power. These images are noisy and the objects of interest are overlaid with other image detail (ducts, veins and glands in mammographic images and skin creases and light reflections in skin images). When the outlines calculated by the algorithm (the "model" outlines) were compared to individual outlines drawn by the subjects for pigmented skin lesions, the model outlines were always within the bounds drawn by the subjects. They tended to be closely related to the "consensus" boundary (defined as having more than 5 votes), with average displacement of 3.3 pixels and standard deviation 2.6. An example is shown in figure 4(d). Comparison of the model outlines with single outlines provided for mammographic lesions showed very good spatial coincidence, with average radial displacement of 2.7 pixels (or 0.67 when the sign of the displacement is noted) and standard deviation 3.6. Moreover, the model outlines, unlike "average" outlines, closely follow undulations of the boundary (figure 4(a)).

In this paper the presented method of edge localisation was contrasted with methods based on zero crossing. It would be interesting to extend comparisons to other, nonlocal, methods, such as active contour models [8].

\section{Conclusions}

The algorithm described in this paper can correctly predict the locations of object boundaries as they would be perceived by human observers. As it is based on general principles believed to underlie the human visual perception it is a general-purpose algorithm which can be applied to images of any objects. Its use would be particularly advantageous for tasks which require the derivation of human-perceived boundaries and where the "ground truth" data is unobtainable. Tedious experiments requiring many subjects to provide the boundary locations for particular image data sets, and subsequent processing of the location data to arrive at a "consensus", can be replaced by the application of the algorithm. Examples of such tasks in 
medical domain include derivation of properties of lesions such as size, shape, shape irregularity etc., and their correlation with a diagnosis (e.g. [15]).

As a further extension of this work, uncertainties associated with boundary placing tasks will be considered. Research in psychophysics has produced semi-quantitative results which allow one to associate parameters of the input data with the magnitude of error. These results could provide objective measures of image quality which can be used, for example, to design viewing conditions which minimise boundary placing errors.

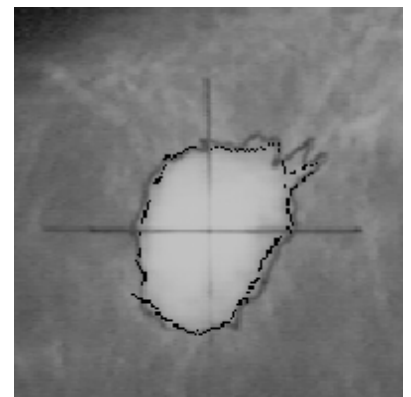

(a)

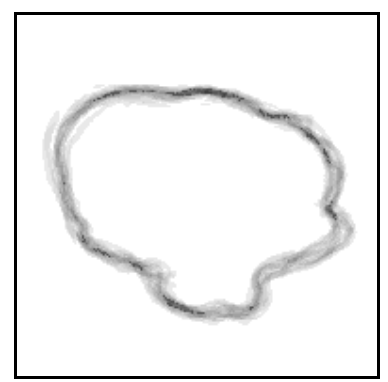

(c)

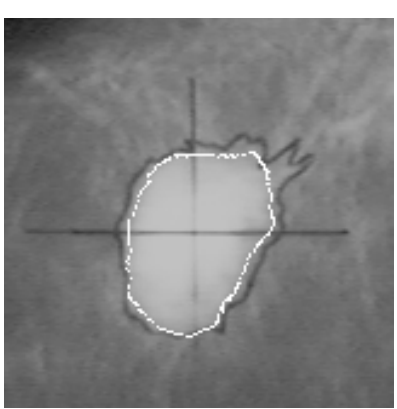

(b)

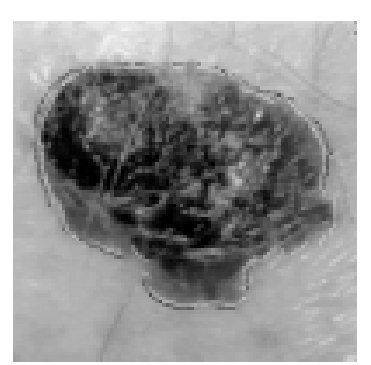

(d)
Figure 4. (a) A mammographic lesion with the edge outlined by a clinician (dark grey) and by the computer algorithm (black); (b) The same lesion, with the edge outlined by a clinician (dark grey) and position of the zero-crossing (white); (c) A combined set of edge locations drawn by the clinicians for a skin lesion shown in (d); the degree of consensus is brightnesscoded (the darker the point the more agreement); (d) A skin lesion with the clinical "consensus" outline (black) and the outline computed by the algorithm.

\section{References}

[1] Claridge E, Jefferies M (1997) The effect of contrast polarity on the shift in perceived edge location in 2D blobs with blurred edges (abstract). Second AVA Christmas Meeting, Aston University, Birmingham, 17 December 1997.

[2] Cotton, S.D., Claridge, E., Hall, P.N. (1997) Noninvasive skin imaging. Information Processing in Medical Imaging, LNCS 1230, 501-507.

[3] Dreher B, Sanderson KJ (1973) Receptive field analysis: Responses to moving visual contours by single lateral geniculate neurons in the cat. Journal of Physiology 234, 95118.

[4] Georgeson M, Freeman TCA (1997) Perceived location of bars and edges in one-dimensional images: computer models and human vision. Vision Research 37, 127-141.

[5] Georgeson MA (1994) From filters to features: location, orientation, contrast and blur. High-order Processing in the Visual System. CIBA Foundation Symposium 184: Wiley \& Sons, 147-169.

[6] Highnam RP, Brady JM, Shepstone BJ (1995) A representation for mammographic image processing. First International Conference on Computer Vision, Virtual Reality and Robotics in Medicine, (Ayache N Ed).

[7] Jefferies M (1997) Perceived location of edges in 2D stimuli: an experiment and computational model. MSc Thesis, Cognitive Science Research Centre, The University of Birmingham.

[8] Kass M, Witkin A, Terzopoulos, D (1987) Snakes: Active contour models. International Journal of Computer Vision, 321-331.

[9] Marr D, Hildreth E (1980) Theory of edge detection. Proceedings of the Royal Society of London B207, 187-217.

[10] Mather G, Morgan MJ (1986) Irradiation: implications for theories of edge localisation. Vision Research 26, 10771015.

[11] Morgan MJ, Mather G, Moulden B, Watt RJ (1984) Intensity-response nonlinearities and the theory of edge localisation. Vision Research 24 713-719.

[12] Petrou M, Kittler J (1991) Optimal edge detectors for ramp edges. IEEE Transactions on Pattern Analysis and Machine Intelligence 13, 483-491.

[13] Richter J, Claridge E (1991) Extraction of quantitative blur measures for circumscribed lesions in mammograms. Medical Informatics 16, 229-240.

[14] Watt RJ, Morgan MJ (1983) The recognition and representation of edge blur: evidence for spatial primitives in human vision. Vision Research 23, 1465-1477.

[15] White RG, Perednia DE Schowengerdt RA (1991) Automated feature detection in digital images of skin. Computer Methods and Programs in Biomedicine 34, 41-60.

[16] Wilson HR, Bergen JR (1979) A four mechanism model for spatial vision. Vision research 19, 19-32. 